\title{
(6) \\ OPEN ACCESS \\ Identifying systems failures in the pathway to a catastrophic event: an analysis of national incident report data relating to vinca alkaloids
}

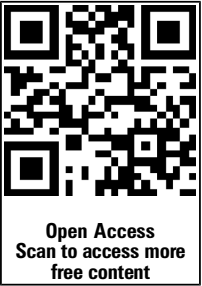

\author{
Bryony Dean Franklin, ${ }^{1,2}$ Sukhmeet S Panesar, ${ }^{3}$ Charles Vincent, ${ }^{4}$ \\ Liam J Donaldson ${ }^{5}$
}

${ }^{1}$ Centre for Medication Safety and Service Quality, Pharmacy Department, Imperial College Healthcare NHS Trust, London, UK

${ }^{2}$ Department of Practice and Policy, UCL School of Pharmacy, London, UK

${ }^{3}$ Department of Primary Care and Public Health, Imperial College London, London, UK ${ }^{4}$ Department of Surgery and Cancer, Imperial College London, London, UK

${ }^{5}$ Institute of Global Health Innovation, Imperial College London, London, UK

\section{Correspondence to} Dr Liam J Donaldson, Chair in Health Policy, Institute of Global Health Innovation, 10th Floor, Queen Elizabeth the Queen Mary Wing (QEQM), St Mary's Campus, Imperial College London, London, W2 1NY, UK; I.donaldson@imperial.ac.uk

Received 7 October 2013 Revised 20 December 2013 Accepted 17 February 2014 Published Online First

18 March 2014

\section{(a) CrossMark}

To cite: Franklin $\mathrm{BD}$, Panesar SS, Vincent $C$, et al. BMJ Qual Saf 2014;23:

765-772.

\begin{abstract}
Background Catastrophic errors in healthcare are rare, yet the consequences are so serious that where possible, special procedures are put in place to prevent them. As systems become safer, it becomes progressively more difficult to detect the remaining vulnerabilities. Using inadvertent intrathecal administration of vinca alkaloids as an example, we investigated whether analysis of incident report data describing low-harm events could bridge this gap.
\end{abstract}

Methods We studied nine million patient safety incidents reported from England and Wales between November 2003 and May 2013. We searched for reports relating to administration of vinca alkaloids in patients also receiving intrathecal medication, and classified the failures identified against steps in the relevant national protocol.

Results Of 38 reports that met our inclusion criteria, none resulted in actual harm. The stage of the medication process most commonly involved was 'supply, transport and storage' (15 cases). Seven cases related to dispensing, six to documentation, and four each to prescribing and administration. Defences most commonly breached related to separation of intravenous vinca alkaloids and intrathecal medication in timing $(n=16)$ and location ( $n=8)$; potential for confusion due to inadequate separation of these drugs therefore remains. Problems involved in six cases did not align with the procedural defences in place, some of which represented major hazards.

Conclusions We identified areas of concern even within the context of a highly controlled standardised national process. If incident reporting systems include and encourage reports of no-harm incidents in addition to actual patient harm, they can facilitate monitoring the resilience of healthcare processes. Patient safety incidents that produce the most serious harm are often rare, and it is difficult to know whether patients are adequately protected. Our approach provides a potential solution.

\section{INTRODUCTION}

The first reports of deaths from the administration of intravenous vincristine via the incorrect route started to appear in the medical literature in the late 1960s. ${ }^{1}$ Despite an awareness of the problems associated with vincristine and other vinca alkaloids for over 45 years, serious and sometimes fatal errors associated with their use still occur. ${ }^{2}{ }^{3}$ Such events remain very rare but have devastating and tragic consequences for the people concerned (often children) and their families.

Investigations of such incidents have shown them to be classic systems failures and thus amenable to prevention. ${ }^{4} 5$ Analyses have found vulnerabilities at every stage of the process, including inadequate labelling, ambiguous procedures, lack of standardisation across units and other hazards. Newer, unexpected failures at the manufacturing stage have also been reported. ${ }^{6}$ In the absence of a long sought-after failsafe involving a new spinal injection connecting system, ${ }^{7}$ the safety of patients receiving intravenous vinca alkaloids as part of cytotoxic chemotherapy continues to depend on compliance with guidelines and protocols.

The challenge of eliminating these tragic events has inspired policy statements from, among others, the North American Institute of Safe Medication Practices, the UK Government, the Australian Council for Safety and Quality 
in Health Care, the Ministry of Health in Hong Kong and WHO. ${ }^{8-11}$ In the UK ${ }^{12}$ and other countries, ${ }^{5} 10$ guidance and protocols have been issued that specify the exact processes required for the administration of intravenous vincristine (and other vinca alkaloids) in patients who are also receiving intrathecal chemotherapy. These policy statements and resulting changes in procedure have done much to reduce the risks of the maladministration of vinca alkaloids. However, given that the adverse outcome is so rare, there is no easy way of identifying any remaining vulnerabilities, or monitoring the current risk to patients.

In the UK, to the best of our knowledge, there have been no deaths or serious incidents since $2001 .^{1}$ The very success of current policies and procedures makes the identification of any remaining vulnerabilities much more challenging. In Amalberti's telling phrase, it is one of the 'paradoxes of ultra-safe systems" ${ }^{13}$; the safer a system becomes, the less safety information is available to monitor current safety or to guide future safety interventions. The deaths from vincristine, while dreadful, led to an opportunity to learn and improve. The challenge is now to monitor and further improve safety in the absence of evidence of harm.

In this study, we use the National Reporting and Learning System (NRLS), a uniquely large database of patient safety incidents from England and Wales, to examine current hazards associated with the administration of vinca alkaloids. NRLS data have previously been used to identify other high-risk, low-frequency, events. ${ }^{14-20}$ Our objectives were to: (1) identify and analyse reports relating to administration of intrathecal medication in patients also prescribed intravenous vinca alkaloids; (2) identify the defences breached and (3) consider the implications for the current and future safety of the systems and processes involved. We also consider the wider usefulness and potential of using very large databases of reported incidents to study rare but potentially serious patient safety events.

\section{METHODS}

\section{Context}

Our analysis was conducted in 2013. At this time, national guidance was in place across England and Wales requiring hospitals to instigate a series of actions relating to the safe intrathecal administration of chemotherapy, and to prevent the administration of vinca alkaloids intended for intravenous administration via the incorrect route. Specifically, Department of Health guidance was initially issued in November $2001^{21}$ to support a national target to reduce to zero the number of patients dying or being paralysed by maladministered spinal injections by the end of $2001 .^{22}$ The aim was to ensure uniform practice across England and Wales so that staff moving from one hospital to another would not have to adapt to a different procedure. This guidance was then updated and reissued in October $2003^{23}$; all English National Health Service (NHS) hospitals providing an intrathecal chemotherapy service were peer reviewed between November 2004 and 2007 to ensure compliance against this guidance. In August 2008, the National Patient Safety Agency (NPSA) issued a Rapid Response Report additionally recommending the use of $50 \mathrm{~mL}$ minibags to dilute vinca alkaloids administered in adult and adolescent units, with an implementation deadline in February 2009. ${ }^{24}$ The Department of Health guidance was then updated in 2008 to take into account NPSA guidance and additional risks highlighted by the peer review process, with an implementation date of the end of December $2008 .^{25}$ Key elements of current guidance are summarised in figure 1.

\section{Data source}

The study population was derived from the database of approximately nine million reports of patient safety incidents reported from the NHS in England and Wales over the last decade. Since 2002, NHS organisations and their staff have been encouraged to report to the NRLS any occurrence of harm or potential harm to a patient as a 'patient safety incident', defined as 'any unintended or unexpected incident which could have, or did, lead to harm for one or more patients receiving NHS care. ${ }^{, 26}$ Prior to March 2012, reports to the NRLS were received, evaluated and acted upon by the NPSA, an independent body at arm's length from the headquarters of the NHS. ${ }^{18}$ Following abolition of the NPSA in $2010,{ }^{27}$ the tasks of evaluating incident reports and issuing alerts to the NHS transferred to NHS England, the body responsible for managing the English NHS.

Each incident report includes structured information in a number of categories: demographic and administrative; the circumstances of occurrence; a categorisation of causation; an assessment of the degree of actual harm ('no', 'low', 'moderate', 'severe', 'death'); and action taken, or planned, to investigate or prevent a recurrence. Additionally, a section of the report allows the staff member making the report to state in free text narrative fashion what happened and why they think it happened. Data are anonymised to remove details that identify any patients or staff. A full description of the NRLS database can be found elsewhere. ${ }^{20} 27$

\section{Reports extracted from the patient safety database}

We searched the free text incident description field of incidents reported over the period 1 November 2003-16 May 2013 for those which included any of the following terms: vincristine, vinca, Oncovin, vindesine, vinflunine, vinorelbine or vinblastine. The output from this search was then subject to a further free text search using the terms 'intrathecal' and 'spinal'. We then read each report retrieved to identify those that met our inclusion criterion of incidents that related to the use of intravenous vinca alkaloids in patients also prescribed intrathecal medication. 
Administration only in designated centres:

(1) Designated lead

(2) Written local protocol, based on national guidance

- All

Only those on local register can prescribe, dispense, issue, check or administer IT chemotherapy:

(1) Induction training, annual competency review

(2) Training grades cannot prescribe

IT chemotherapy on separate prescription with accompanying checklist and audit trail

- Prescribing

- Documentation

IV and IT chemotherapy separated in time

(1) Administered at separate times - IV then IT

(2) Signature to confirm all IV chemo for that day given, before IT released to the doctor who will administer it

IV and IT chemotherapy separated in location

(1) Separate storage in pharmacy and ward areas, with IT doses in a dedicated locked fridge

(2) Separate transport of IT in distinctive container

(3) Administration in separate clinical areas

IV \& IT chemotherapy differentiated in appearance:

(1) IV vinca alkaloids for adults and adolescents prepared in minibags, not syringes

(2) Labelling of medication with route of administration printed in bold "for intrathecal use only" and "for intravenous use only"

Under normal circumstances, administered during working hours only. Authorisation and reporting of any exceptions

- $\quad$ Prescribing

- Dispensing

- Administration

Administration checks:

(1) Documented patient review prior to IT administration

(2) Documented second check by separate member of staff on local register, using formal checking procedure

(3) Involve patient in checks if he/she chooses to do so

- Prescribing

- Supply, transport and storage

- Administration

- Documentation

- Dispensing

Figure 1 Summary of defences to prevent errors in the administration of intrathecal chemotherapy, based on Department of Health guidance on the safe administration of intrathecal chemotherapy. ${ }^{25} \mathrm{IT}$, intrathecal; IV, intravenous.

\section{Analysis}

We identified the degree of harm reported, and the year in which the incident occurred. We then identified the principal stage of the medication use process in which the patient safety incident occurred, according to the following schema:

prescribing

- dispensing (preparation and labelling of the medication in the pharmacy)

- supply, transport and storage (including release of medication from the pharmacy)

- administration of medication to the patient

- documentation (including documentation of medication administration and the associated audit trail).
Finally, we identified from the free text descriptions, the principal defence(s) according to figure 1 that had been breached for each incident.

Coding was carried out independently by two researchers (BDF and SP), and any discrepancies resolved by discussion.

\section{RESULTS}

\section{Overview of incident reports}

Our search strategy initially revealed 44 reports. Of these 44, six concerned patient safety incidents that involved other drugs or procedures and, therefore, did not meet our inclusion criteria. For example, one 
concerned the omission of pegylated aspariginase in a patient who was also receiving doxorubicin and vincristine as part of their chemotherapy protocol; another concerned the omission of folinate in a patient-prescribed vincristine and cyclophosphamide. These six cases were therefore excluded from further analysis, leaving 38 remaining reports. Examples of two of these are presented in box 1 .

A further two of the 38 reports concerned intrathecal antibiotics (rather than intrathecal chemotherapy) being given on the same day and/or being stored in the same location as intravenous vinca alkaloids. While not covered by the Department of Health guidance, ${ }^{25}$ these were felt by staff at the organisation concerned to represent a similar risk. We thus included these incidents in our initial results but excluded them from our analyses according to the stage of medication use in which the patient safety incident occurred and the defences breached.

All incidents occurred in 2005 or later, with peaks in 2006/2007 (six incidents) and in 2010/2011 (eight incidents). With such small numbers it was not possible to conduct formal analysis of any trends in reported incidents over time, but there was no discernible trend since 2005 .

\section{Impact of incidents on patients}

Of the 38 included reports, 33 were classified by the reporter as resulting in 'no harm', and five as 'low harm'. However, on closer inspection of the descriptions provided, there was no evidence of any actual harm in the five 'low harm' cases. None of the incidents, therefore, appeared to have caused any actual patient harm.

\section{Stage of medication use process}

We excluded the two reports concerning intrathecal antibiotics, plus a further incident for which the description had insufficient detail to determine the stage involved. In one of the remaining 35 cases, two stages were involved (box 1). Overall, the stage of the medication use process most commonly involved was 'supply, transport and storage' (15 cases). Seven cases related to dispensing, six to documentation and four each to prescribing and medication administration.

\section{Defences breached}

After excluding the two antibiotic-related reports and the report for which the description was insufficient, the most common categories of defence breached were those that related to separation of intravenous vinca alkaloids and intrathecal medication in terms of timing $(n=16)$ and location $(n=8)$. Table 1 presents in more detail the specific defences involved. The principal remaining risk appears to be the potential for inadequate separation of intrathecal and intravenous chemotherapy, whether in time (administration on the
Box 1 Examples of cases analysed. Date of incident, date of reporting, ward names and organisational characteristics removed to preserve anonymity

Case 1: detailed description; two stages of the medication use process involved ('supply, transport and storage' and 'documentation')

CATEGORY: Medication

DEGREE OF HARM: No harm

AGE AT INCIDENT: (Not stated)

LOCATION: Inpatient areas

SPECIALTY: Medical specialties/medical oncology

INCIDENT DESCRIPTION: '(1) patient prescribed RCHOP [regimen] chemotherapy by Oncology, and separately prescribed intrathecal methotrexate by Haematology. Two separate requests were received in pharmacy on different days without any communication that the patient was to have both treatments on the same day. Due to the volume of work and staff shortages within the lab, the two requests were not tied together. Consequently the IV chemotherapy was released to [oncology ward] and the intrathecal chemotherapy to [haematology day unit] by different pharmacists at different times without either being aware that the two separate requests existed. This meant that vincristine for this patient was on the ward while the intrathecal was in progress and without confirmation that IV chemotherapy has been given first as per the National Guidance. In the event, the chemotherapy was not given and was returned to pharmacy which was when the incident came to light. (2) during the course of the morning the original addressograph was changed (ie surname / Christian name changed round), after pharmacy had labelled the intrathecal methotrexate, which was then returned to be relabelled'.

Case 2: minimal detail available; stage of medication use process involved 'supply, transport and storage'

CATEGORY: Medication

DEGREE OF HARM: No harm

AGE AT INCIDENT: (Not stated)

LOCATION: Inpatient areas

SPECIALTY: Medical specialties/clinical haematology INCIDENT DESCRIPTION: 'Syringe of vincristine (a dose banded syringe) found in the cytotoxic fridge on the day that the patient was given intrathecal chemotherapy'.

same day) or location (storing or transporting them together).

The six cases classified as 'other' involved those where none of the defences in figure 1 seem to have been breached specifically. These included: two prescribing errors involving the prescription of intrathecal vincristine' which were identified and rectified; a dose of intravenous vinca-based chemotherapy not made for the day required; a patient documented on a theatre list as requiring 'intrathecal vincristine' (rather 
Table 1 Incidents presented according to the main defence (as in figure 1) breached

\begin{tabular}{|c|c|}
\hline Defence & $\begin{array}{l}\text { Number of } \\
\text { reports }\end{array}$ \\
\hline Administration only in designated centres & 0 \\
\hline $\begin{array}{l}\text { Only those on local register can prescribe, dispense, issue, } \\
\text { check or administer intrathecal (IT) chemotherapy }\end{array}$ & 0 \\
\hline $\begin{array}{l}\text { IT chemotherapy on separate prescription with } \\
\text { accompanying checklist and audit trail }\end{array}$ & 1 \\
\hline Intravenous (IV) and IT chemotherapy separated in time & 15 \\
\hline 1. Administered at separate times_IV then IT & 6 \\
\hline $\begin{array}{l}\text { 2. Signature to confirm all IV chemotherapy for that day } \\
\text { given, before IT released to the doctor who will } \\
\text { administer it }\end{array}$ & 9 \\
\hline IV and IT chemotherapy separated in location & 7 \\
\hline $\begin{array}{l}\text { 1. Separate storage in pharmacy and ward areas, with } \\
\text { IT doses in a dedicated locked fridge }\end{array}$ & 5 \\
\hline $\begin{array}{l}\text { 2. Separate storage in pharmacy and ward areas, with } \\
\text { IT doses in a dedicated locked fridge }\end{array}$ & 5 \\
\hline 3. Separate transport of IT in distinctive container & 1 \\
\hline 4. Administration in separate clinical areas & 1 \\
\hline IV and IT chemotherapy differentiated in appearance & 6 \\
\hline $\begin{array}{l}\text { 1. IV vinca alkaloids for adults and adolescents } \\
\text { prepared in minibags, not syringes }\end{array}$ & 1 \\
\hline $\begin{array}{l}\text { 2. Labelling of medication with route of administration } \\
\text { printed in bold 'for intrathecal use only' and 'for } \\
\text { intravenous use only' }\end{array}$ & 5 \\
\hline $\begin{array}{l}\text { Under normal circumstances, administered during working } \\
\text { hours only }\end{array}$ & 0 \\
\hline Administration checks & 0 \\
\hline Other & 6 \\
\hline TOTAL & 35 \\
\hline
\end{tabular}

Bold numbers represent main categories; italic numbers represent subcategories of these.

than intrathecal methotrexate as intended); a missing syringe of intravenous vincristine; and a patient who had the incorrect cannula inserted for the dose of intravenous vincristine required.

The two independent coders initially disagreed on only two occasions: (1) whether or not one of the initial 44 reports retrieved met our inclusion criteria; (2) regarding the stage of the medication use process involved for one of our 38 included incidents. Both were resolved following discussion.

\section{DISCUSSION}

\section{Key findings}

We identified 38 patient safety incidents relating to vinca alkaloids and intrathecal medication that had been reported to a national patient safety database over a 10 -year period. The stage of the medication use process most commonly involved was 'supply, transport and storage', and the defences most commonly breached were those relating to separating intravenous and intrathecal chemotherapy in time and location. None of the reports included any evidence of actual patient harm. There was no clear pattern of incident reporting over time; the absence of reports pre-2005 is likely to reflect the fact that the NRLS was still in development and not yet fully established as a national presence. ${ }^{20} 28$

\section{Interpretation}

Inadvertent intrathecal administration of vinca alkaloids intended for intravenous use is now a very rare event. The NRLS has revealed no reports of harm since 2003. However, the rarity of this event should not be a reason for complacency; first, because further cases occurred internationally in $2011,{ }^{23}$ and second, because continuing safety requires ongoing vigilance and ongoing assessment of current and future risks. In healthcare, our understanding of safety has traditionally relied on the analysis of serious and harmful incidents; however, where systems have become much safer we need to take a more proactive approach and assess more subtle signs of system vulnerability. ${ }^{29}$

The present study, together with the changing healthcare landscape, suggests a number of areas that should be reviewed. First, our study suggests that there is still potential for confusion due to inadequate separation of intravenous and intrathecal drugs. This is particularly worrying because the failure to separate these drugs has played a major role in previous tragedies $^{30}$ and because the separation of drugs upstream in the process is likely to be a more reliable defence than checks by staff at a later stage. Second, more complex drug regimes are constantly emerging which may pose new challenges. For instance, some chemotherapy regimens require chemotherapy to be given on two consecutive days, but intrathecal chemotherapy only given on the first day. This may lead to improvised local rules as the national guidance is based around the intravenous medication being given first. Any ambiguity in such processes puts patients at risk. Third, these findings suggest more general lessons for the administration of other intrathecal drugs. For instance, there is considerable potential for maladministration of medication in patients prescribed concomitant intrathecal antibiotics and intravenous medication, but guidelines are less stringent or not in place at all. Guidance may also be more difficult to implement as intrathecal antibiotics may be given in parallel with a very wide range of other drugs, as well as with intravenous chemotherapy. A solution based on redesign is still needed, as pointed out by Berwick some 12 years ago. ${ }^{31}$

Of particular concern was our finding that the problems involved in six cases did not align with the procedural defences at all, and at least some represented major hazards. It may seem absurd to think that a patient listed for theatre as requiring 'intrathecal vincristine' would actually receive the drug via this fatal route. Yet it has previously proved a folly to believe that common sense or technical knowledge would act as a failsafe. Perhaps, there is even more 
danger if staff believe they are insulated from error because they have correctly implemented and followed recommended procedures.

Of note, none of the incidents analysed referred to electronic prescribing (EP), and yet chemotherapy EP systems are now used in 34\% of English hospitals. ${ }^{32}$ It may be that intrathecal medication is not yet being prescribed electronically; local experience suggests that some EP systems do not support the prescribing and audit trail requirements, resulting in the potential for intrathecal chemotherapy to be prescribed on paper, and other chemotherapy to be prescribed electronically. Such split prescribing practices would potentially pose new hazards.

\section{Strengths and limitations}

The NRLS is one of the few databases with the capacity to identify rare events of the kind studied here. Its size, duration and the inclusion of reports of low levels of harm as well as adverse outcomes, are all unique strengths. The main limitation is that there may be considerable under-reporting of incidents concerning vinca alkaloids, just as for other issues. ${ }^{20}$ Nevertheless, of all the errors in healthcare, the catastrophic nature of the maladministration of vincristine has meant that it has featured as an example in policy documents $^{22}$ and training materials. ${ }^{34}$ NHS staff are likely to have a high level of concern about any incidents of this type, a phenomenon Reason has termed 'chronic anxiety'. ${ }^{35}$ Our analysis is limited to what has been reported on the system, in terms of accuracy and the level of detail. The quality of the reports varies widely (box 1), although this has not formally been investigated. ${ }^{28}$ The sample of incidents is also valuable as it provides information about vulnerabilities of the system. It was beyond the scope of our study to identify the ways in which violations of procedures were recognised and corrected, and the reports generally included limited detail of the reporter's interpretation of the causes or the actions taken as a result.

\section{Implications for practice}

To be able to anticipate risks in the absence of harm is the hallmark of a resilient system. ${ }^{33}$ It points to a more proactive approach to safety in healthcare which, in turn, relies on the integration and analysis of diverse sources of safety information. ${ }^{29}$ The original report which provided the foundation for patient safety in the UK NHS was called An Organisation with a Memory, precisely because it sought to provide a means of learning from incidents across the entire system. Isolated tragedies occurred constantly but, because no connections were made, no collective action was taken. When systems become safer, this challenge is even greater. How can we identify small threats to patients across a system as large as the NHS? The true value of large-scale reporting systems is not in the accumulation of vast numbers of reports but in the identification of rare or unusual events scattered across the wider system which might otherwise escape notice and put patients at risk. ${ }^{22} 3637$ We would therefore recommend that consideration be given to how incident reporting systems can best be used to identify risks of the type identified here. Other high-risk incidents, such as those relating to concentrated potassium chloride, could also be studied in this way. At present, requests for data can be made to NHS England, the body responsible for commissioning healthcare for people living in England. Such requests are currently granted to bona fide organisations on a case-by-case basis. However, making properly anonymised incident report data more readily available to researchers, professional bodies, clinicians and patient representatives would provide further opportunities for learning.

We have also identified specific risks which appear to remain, concerning the separation of intravenous and intrathecal chemotherapy in time and space, and highlighted some areas where further action may be needed, such as in the area of intrathecal antibiotics.

Of concern is that some incident reports were lacking detail and did not give a full account of the nature of the hazard. Encouraging more comprehensive narratives when reporting is as important, arguably more so, than increasing reporting rates.

\section{CONCLUSION}

We identified 38 patient safety incidents relating to vinca alkaloids and intrathecal medication reported over a 10-year period. Problems most commonly related to separating intravenous and intrathecal chemotherapy in time or location. In the UK, to the best of our knowledge, there have been no deaths or serious incidents associated with the spinal injection of vinca alkaloids in recent years. Yet, in other fields of risk, such as aviation, the absence of an accident is not an acceptable basis to conclude that a system is safe. The deaths from maladministration of vincristine, while dreadful, afforded the opportunity to learn and improve. The challenge, across all areas of harm, is now to create and use data on upstream low-harm occurrences to test the resilience of safety practices and systems. We here suggest how this may be done using large incident report databases to study risks related to rare but very serious adverse events.

Acknowledgements We thank David Leonard, Executive Lead Pharmacist (Technical Services) at Imperial College Healthcare NHS Trust for his helpful comments on a draft manuscript.

Contributors The study was conceived by LD and CV with all four authors contributing to study design. Data were retrieved by SP, and analysed by BDF and SP. BDF led the writing of the paper together with the other authors.

Funding This work was part of the ongoing research undertaken at the National Reporting and Learning System, and no extra funding was sought The CMSSQ is affiliated with the Centre for Patient Safety and Service Quality (CPSSQ) at Imperial College Healthcare NHS Trust which is funded by the 
National Institute of Health Research as a Patient Safety Translational Research Centre.

\section{Competing interests None.}

Ethics approval This study was part of a research programme funded at Imperial College by NHS England to develop incident reporting in the NHS. Ethical approval is in place for this programme from the Health Research Authority.

Provenance and peer review Not commissioned; externally peer reviewed.

Open Access This is an Open Access article distributed in accordance with the Creative Commons Attribution Non Commercial (CC BY-NC 3.0) license, which permits others to distribute, remix, adapt, build upon this work noncommercially, and license their derivative works on different terms, provided the original work is properly cited and the use is non-commercial. See: http://creativecommons.org/licenses/by$\mathrm{nc} / 3.0 /$

\section{REFERENCES}

1 Noble DJ, Donaldson LJ. The quest to eliminate intrathecal vincristine errors: a 40-year journey. Qual Saf Health Care 2010;19:323-6.

2 Reddy GK, Brown B, Nanda A. Fatal consequences of a simple mistake: how can a patient be saved from inadvertent intrathecal vincristine? Clin Neurol Neurosurg 2011;113:68-71.

3 Pongudom S, Chinthammitr Y. Inadvertent intrathecal vincristine administration: report of a fatal case despite cerebrospinal fluid lavage and a review of the literature. J Med Assoc Thai 2011;94(Suppl 1):S1-6.

4 Toft B. External inquiry into the adverse incident that occurred at Queen's Medical Centre, Nottingham, 4 January 2001. http://www.who.int/patientsafety/news/Queens\%20Medical\% 20Centre\%20report $\% 20$ (Toft).pdf

5 Tannock I. Report on a medication incident of Intrathecal administration of vincristine in Prince of Wales Hospital. 2007. http://www.ha.org.hk/investigation_panel/pwh/report_eng.pdf

6 Kurtenback E. China shuts down leukemia drug maker. The Associated Press, 2007. http://www.usatoday.com/news/health/ 2007-12-12-1698973141_x.htm

7 National Patient Safety Agency. Minimising risks of mismatching spinal, epidural, and regional devices with incompatible connectors. 2011. http://www.npsa.nhs.uk/ corporate/news/minimising-risks-spinal-devices/

8 ISMP Canada Safety Bulletin. Epidural medications given intravenously may result in death. 2006. http://www. ismp-canada.org/download/safetyBulletins/ ISMPCSB2006-07Epidural.pdf

9 Department of Health UK. The prevention of intrathecal medication errors: a report to the Chief Medical Officer. 2001. http://webarchive.nationalarchives.gov.uk/+/www.dh.gov.uk/en/ Publicationsandstatistics/Publications/ PublicationsPolicyAndGuidance/DH_4065044

10 Australian Council for Safety and Quality in Health Care. Key achievements of the Australian Council for Safety and Quality in Healthcare. MJA 2006;184:S37.

11 World Health Organization. Vincristine sulphate. 2007. http:// www.who.int/patientsafety/activities/technical/vincristine/en/ index.html

12 National Patient Safety Agency. Vinca alkaloid minibags. 2008. http://www.nrls.npsa.nhs.uk/resources/?entryid45=59890

13 Amalberti R, Auroy Y, Berwick D, et al. Five system barriers to achieving ultrasafe health care. Ann Intern Med 2005;142:756-64.
14 Lamont T, Beaumont C, Fayaz A, et al. Checking placement of nasogastric feeding tubes in adults (interpretation of $\mathrm{x}$ ray images): summary of a safety report from the National Patient Safety Agency. BMJ 2011;342:d2586.

15 Lamont T, Dougall A, Johnson S, et al. Reducing the risk of retained swabs after vaginal birth: summary of a safety report from the National Patient Safety Agency. BMJ 2010;341: c3679.

16 Healey F, Sanders DS, Lamont T, et al. Early detection of complications after gastrostomy: summary of a safety report from the National Patient Safety Agency. BMJ 2010;340:c2160.

17 Lamont T, Watts F, Stanley J, et al. Reducing risks of tourniquets left on after finger and toe surgery: summary of a safety report from the National Patient Safety Agency. BMJ 2010;340:c1981.

18 Lamont T, Scarpello J. National Patient Safety Agency: combining stories with statistics to minimise harm. BMJ 2009;339:b4489.

19 Rocos B, Donaldson LJ. Alcohol skin preparation causes surgical fires. Ann R Coll Surg Engl 2012;94:87-9.

20 Panesar SS, Salvilla SA, Patel B, et al. Laparoscopic cholecystectomy: device-related errors revealed through a national database. Expert Rev Med Devices 2011;8:555-60.

21 Department of Health. HSC 2001/022: National guidance on the safe administration of intrathecal chemotherapy. 2001. http://webarchive.nationalarchives.gov.uk/+/www.dh.gov.uk/en/ Publicationsandstatistics/Lettersandcirculars/

Healthservicecirculars/DH_4004769

22 Department of Health. An Organisation With a Memory. 2000. http://webarchive.nationalarchives.gov.uk/+/www.dh.gov. uk/en/Publicationsandstatistics/publications/ publicationspolicyandguidance/browsable/dh_4098184

23 Department of Health. HSC 2003/010: Updated national guidance on the safe administration of intrathecal chemotherapy. 2003. http://webarchive.nationalarchives.gov.uk/ +/www.dh.gov.uk/en/Publicationsandstatistics/ Lettersandcirculars/Healthservicecirculars/DH_4064931

24 National Patient Safety Agency. Rapid Response Report 4: Vinca alkaloid minibags (adult|adolescent units). 2008. http:// www.nrls.npsa.nhs.uk/resources/type/alerts/? entryid $45=59890 \& \mathrm{p}=2$

25 Department of Health HSC 2008/001: Updated national guidance on the safe administration of intrathecal chemotherapy. 2008. http://webarchive.nationalarchives.gov.uk/ +/www.dh.gov.uk/en/publicationsandstatistics/ lettersandcirculars/healthservicecirculars/dh_086870

26 National Patient Safety Agency. Seven Steps to Patient Safety. 2004. http://www.nrls.npsa.nhs.uk/resources/? entryid $45=59787$

27 NHS Commissioning Board. Transfer of patient safety functions to the NHS Commissioning Board. 2012. Available online at http://www.commissioningboard.nhs.uk/2012/05/31/ npsa-transfer/

28 National Patient Safety Agency. NRLS Quarterly Data Workbook up to March. 2012. http://www.nrls.npsa.nhs.uk/ resources/collections/quarterly-data-summaries/? entryid45 $=135152$

29 Vincent C, Burnett S, Carthey J. The measurement and monitoring of safety. The Health Foundation, 2013. http:/ www.health.org.uk/publications/ the-measurement-and-monitoring-of-safety/

30 Catalogue of blunders that led to death. BBC 2001. http:// news.bbc.co.uk/1/hi/health/1284244.stm 
31 Berwick DM. Not again! Preventing errors lies in redesign not exhortation. BMJ 2001;322:247-8.

32 Ahmed Z, McLeod M, Barber N, et al. The use and functionality of electronic prescribing systems in English acute NHS trusts: a cross-sectional survey. PLoS ONE 2013;8:e80378.

33 Chassin MR, Loeb JM. The ongoing quality improvement journey: next stop, high reliability. Health Aff (Millwood) 2011;30:559-68.

34 World Health Organization. Learning from Error-video and booklet. 2008. http://www.who.int/patientsafety/education/ vincristine_download/en/
35 Reason J. Patient safety, human error, and Swiss cheese. Interview by Karolina Peltomaa and Duncan Neuhauser. Qual Manag Health Care 2012;21:59-63.

36 Thomas MJ, Schultz TJ, Hannaford N, et al. Mapping the limits of safety reporting systems in health care-what lessons can we actually learn? Med J Aust 2011;194: 635-9.

37 Panesar SS, Cleary K, Sheikh A. Reflections on the National Patient Safety Agency's database of medical errors. J R Soc Med. 2009;102:256-8. 